\title{
InsBot : Design of an Autonomous Mini Mobile Robot Able to Interact with Cockroaches
}

\author{
Alexandre Colot, Gilles Caprari and Roland Siegwart \\ Autonomous Systems Lab (http://asl.epfl.ch) \\ Swiss Federal Institute of Technology Lausanne (EPFL) \\ CH-1015 Lausanne, Switzerland \\ (alexandre.colot; gilles.caprari; roland.siegwart)@epfl.ch
}

\begin{abstract}
This paper presents general ideas on design and implementation of robots to be used together with groups of animals. This work is part of the European project LEURRE aiming to study, model and control mixed societies of animals and robots. After a short presentation of the general project, the implications on the special requirements will be discussed. The design process of the robot will be described leading to the implementation of the autonomous mini robot called InsBot. This is very compact $(19 \times 30 \times 41 \mathrm{~mm} 3)$, has many sensors (12 IR proximity, linear camera and temperature) and is able to interact with gregarious cockroaches.
\end{abstract}

\section{INTRODUCTION}

Interaction between robots and animals in mixed societies is really a big challenge and an absolutely new research field. During many years researchers over the world have developed robots that are mechanically inspired by animals $[1][2][3][4][5]$ or robots that uses biologic actuators [6] but only few robots that interacts with animals [7][8] and none which tries to be accepted in the society as another animal. This kind of research is basis for further research that can be applied in agriculture and maybe one day for a better interaction with the most sophisticated animal: humans.

The LEURRE European project, started in October 2002, has precisely these goals. In LEURRE, researchers from Belgium (Université libre de Bruxelles ULB, International Solvay Institutes for Physics and Chemistry ISI), France (CNRSEVE at Université de Rennes 1, CNRS-CRCA at Université Paul Sabatier) and Switzerland (Swiss Federal Institute of Technology in Lausanne) are working on different mixed societies of animals and robots, and try to make them interact. For the biologist point of view, it will help to understand easily animal behaviours because of the possibility to program and test behaviours on artificial systems. Whereas for engineers, this is first a challenge of building very small robots that can be compatible with animals. Secondly it is of interest to study perception and sensors for bio-interaction. Finally the behaviour aspects are very important for collective robotics.

The exact goals of LEURRE European project are described bellow:

- Behavioural model. We will propose a formal behavioural model, which applies to mixed societies, and study its properties. We will formalize the behaviours in a programming language and provide a library of tools for dealing with them. All of this will be stored in a behaviour database with appropriate user-friendly interfaces.

- Interpretations and "real" worlds: the mixed-societies. We will provide a validation of the behavioural model -i.e., show that it gives an understanding of the computational capabilities of animal societies - by confronting two of its realizations: on the one hand a realization is given through simulations on computers; on the other hand, another realization is in a concrete and "real" mixed society of insects and insect-like robots.

- Controlling the global behaviour of the society. We will control mixed societies. We will show that it is actually feasible to change the global behaviour of a mixed society and a demonstration will be provided on our "real" mixed society.

- Towards some general methodology. We will provide a general methodology for the study and control of mixed societies. For instance we will answer such questions, as "are there typically configuration patterns that support a priori behavioural organization of mixed societies".

- Relevance of our results to quality of life and management of living resources. The evidence of the relevance of our results to other configurations of mixed-societies (in agriculture) will be provided. Real-Life agricultural experiments will be performed to the endeavour.

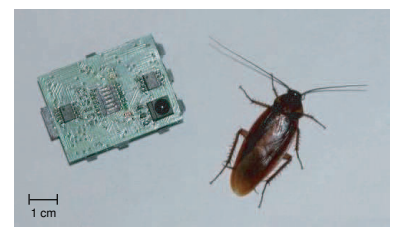

Fig. 1. InsBot (Insect-Robot) and Cockroach.

In this project, the EPFL Team is mainly involved in designing and building the robots (Fig. 1) and of course all tools that are needed to work efficiently with them. For this reason the paper will mainly address the design of the robot while leaving to other publications the other aspects (interaction, results, behaviours) of LEURRE. Another important EPFL task is to program the behaviours according to the models developed by the biologists and to fit them on the robot's $\mathrm{CPU}$, taking into account the hardware limitations. Our last field of research is automatic analysis systems like tracking 
software to decrease the time used after each experiment to analyse the parameters of the behaviour of each animal. In short we are involved in building something that can be used as a toolbox for ethologists.

\section{REQUIREMENTS FOR INSBOT}

As discussed before, we want to develop robots that:

- behave like the real animals of the mixed society.

- are accepted by other animals as a congener.

- are able to influence the global behaviour of the society.

The aim is to study societies of animals and thus a large group of individuals should be used. Moreover, as this research is somehow at the beginning it is convenient to use simple animals permitting a detailed analysis and a reasonable modelling. For these reasons insects with gregarious behaviours are good candidates (size of the experimental setup, animal price, etc.). To better understand the InsBot requirements, we first have to introduce some information about the exact species that has been selected. The typical experimental setup has also consequences on the robot's needs.

\section{A. Cockroach specie and behaviour}

The first mixed-society chosen is InsBots-Cockroaches.

1) Periplaneta Americana: This is a large cockroach (24$44 \mathrm{~mm}$ long) (Fig. 2-3), shining red-brown which is a domiciliary species. It has 6 legs for locomotion, and two long antennas (around $3 \mathrm{~cm}$ ) that are used as tactile sensors.

This gregarious insect has been studied a lot [9] and it doesn't seem to have any active chemical communication [10]. Pheromones are used only for congener recognition so there is no need of any chemical emission and any chemical sensitive system. For our experiment only males will be used to avoid any sexual behaviours and thus the chemistry is less an issue. A deeper description of this insect would be out of the scope of this paper, but further information can be found in the literature.

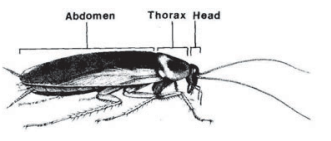

Fig. 2. Male Periplaneta Americana

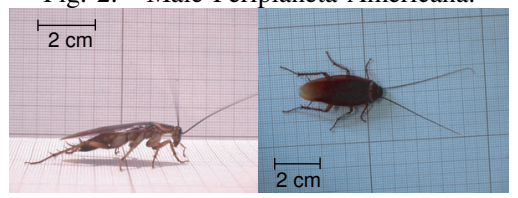

Fig. 3. Side and Top view of the selected cockroach.

2) Individual Behaviour: The individual behaviour is quite simple (Fig. 4), in an empty space the behaviour is nothing else then a Random-Walk, whereas near the wall this is tigmotactism (Wall-Following). It will be modelled with a statistical approach.

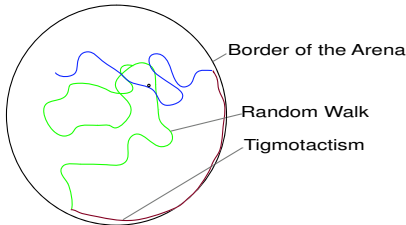

Fig. 4. Individual Behaviour: track of single insect in a circular arena.

3) Collective Behaviour: The gregarious behaviour is just a little bit more complicated. It can be modelled with the individual behaviour plus a probability to stop correlated to the number of individuals already stopped in a local area of perception [11] (Fig. 5). This is something really important, indeed the collective behaviour of the society can be explained by just simple individual behaviours [12][13][14].

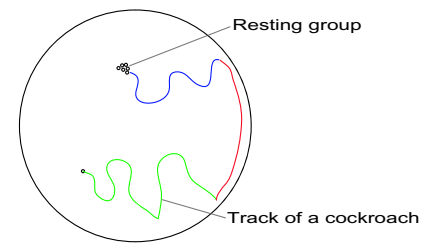

Fig. 5. Collective Behaviour: an individual behaves as being alone before meeting a group of congeners, there it stops.

4) Influence of the InsBot: The robot can modify the general behaviour of the mixed society and can be measured in such a case where the probability to have an aggregate in each shelter would be otherwise equal (Fig. 6). One experimental idea is to influence the aggregate position by upgrading the individual behaviour of the InsBots.

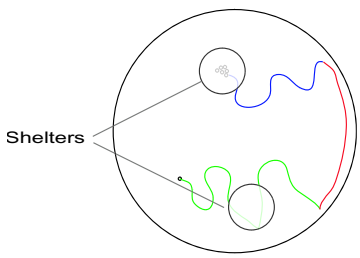

Fig. 6. Influence on society: the InsBots will modify the preferences of the cockroaches to be in one place instead of the other.

5) Models: Models of these behaviours will be extracted statistically from insect's behaviours. That's why we have first developed a tracking system to extract the positions $\left(X_{i}, Y_{i}\right.$, Time $\left._{i}\right)$ for each object in the setup. Then another software will extract all probabilities distributions (angle rotation distributions, segment length, etc.). For example after an analysis of the stopped time, it has been detected that the distribution was really distinctive (something like two Gaussian distribution), and biologist finally found that the stop time is really different if the cockroach detects a congener or an object. So we will use this method to detect if our robot is considered as just an object or as a congener.

6) Experimental Setup: It (Fig. 7) is composed of a white plastic arena (1 $\mathrm{m}$ diameter and $15 \mathrm{~cm}$ high), a top camera and a neon light. A special light has been chosen because of its 


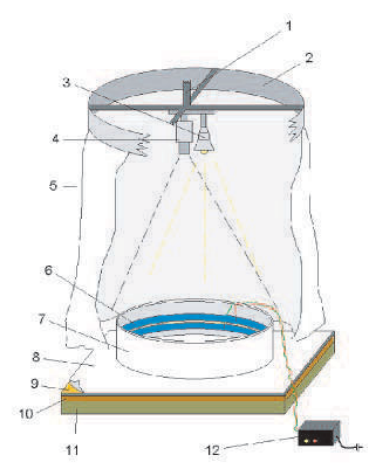

Fig. 7. Experimental Setup is composed by many parts: neon light (3), top camera (4), electrical barrier (6), white plastic arena (7), paper layer (8), wood layer(10), phonic layer (11).

very low infrared emission that would cause some problems for the cockroaches and the IR proximity sensors of the robot. Cockroaches tend to stay in group but when the environment is getting warmer or noisy, cockroaches are much agitated and sometimes they try to climb over the arena and escape. For ecological reasons, we have to avoid that they escape from the arena and are introduced in the environment, so an electrical barrier has been added. This is absolutely not harmful for the cockroaches. The first experiments have shown that vibrations could scare the cockroaches (human and robot vibrations), that's why a phonic layer has been added between the ground and the wood layer. On the wood layer, a paper layer is added and changed after each experiment. This, to avoid any chemical marks that could perturb the next experiments and to remove cockroach's dust that could give troubles to the mechanical parts of the robots because their very small opened watch motors are sensitive to dust.

During the experiment there are different phases: the first is to introduce the animals and robots in the arena and let them reduce their stress around 30 minutes. During this time the robots will be in stand-by mode. After this first phase, the experiment can start and at this point there are many interactions and movements of the cockroaches in the arena. The first experiments done without robots have shown that it takes around 2 hours before the appearance of an aggregation. So the mean experiment time will be around 3 hours.

\section{B. Conclusions about requirements}

Considering the description of the cockroaches and how the experiments will be done, the most difficult points are: the necessary small size of the robot, the high level of integration (many sensors) and the darkness of the cockroach's shell (IR sensors sensitivity). The number and duration of the experiments impose to develop reliable robots and tools.

However the behaviours to be programmed seem to be not too sophisticated and thus feasible with a simple microcontroller architecture. Even if the robots must be very small and compact, a smaller robot (Alice) has already been developed in our lab giving us the knowledge on such systems. Finally, the most important information for all behaviours is proximity which is well managed in robotics.

\section{DESIGN}

During the first part of the project we have used Alice robots [15] to conduct some acceptance tests which reveal that cockroaches were absolutely not afraid by the robot in particular regarding to IR emission, vibration and size.

After these preliminary tests we verified that it was quite hard to detect the cockroaches because of their brown colour which absorb all IRs so we had to power a little bit more the emitters. We also found that we needed some wireless communication modules for monitoring, some other sensors, more computational power and much more memory. That's why we finally decided to develop a new robot specific for our mixed-society application.

\section{A. Discussion about sensors}

Many sensors might be interesting to interact with animals, here is a short abstract of what we had imagined. In this table each sensor has been evaluated on different aspects: computational power, energy consumption and complexity from 1 to 6 (TABLE I).

Because of the limitations on the size of the robot, the

TABLE I

SENSORS TYPES FOR "BIO-ROBOTS"

\begin{tabular}{|l|l|c|c|c|}
\hline $\mathrm{N}$ & Type & Comput. Power & Energy & Complexity \\
\hline 1 & IR proximity & 2 & 3 & 1 \\
\hline 2 & Light sensor & 1 & 1 & 1 \\
\hline 3 & 2D colour camera & 6 & 5 & 6 \\
\hline 4 & Linear camera & 3 & 4 & 3 \\
\hline 5 & Tactiles antennas & 1 & 1 & 4 \\
\hline 6 & Vibration sensor & 1 & 1 & 3 \\
\hline 7 & Temperature & 1 & 2 & 1 \\
\hline 8 & Chemical & 4 & 3 & 6 \\
\hline 9 & Humidity & 1 & 2 & 2 \\
\hline 10 & Gas sensor & 1 & 2 & 2 \\
\hline
\end{tabular}

autonomy and the computational power, we finally decided to implement sensors $1,2,4$ and 7 in the InsBot.

\section{B. Sensors choice}

1) Chemical Sensors: The most important sensors would be chemical sensors. But these are still a major research field and there are currently no industrial sensors that can be found. That's why we decided to use only a passive chemical communication with just a piece of paper impregnated with the synthesized cuticle pheromones of the cockroach. This piece of paper is placed on the robot so that the cockroaches feel the presence of a congener and not only a piece of hardware. On the other way the robot sense the cockroaches not by chemistry but with the IR proximity sensors.

2) IR Sensors: The IR sensors will be used for both proximity and brightness measurement. This is the most important sensor, because every behaviour is mainly based on the proximity information.

Not only the kind of sensors is important but also position of sensors itself. They have been specially positioned in order to 
make a difference between a wall and a cockroach. That's why we decided to place one sensor on top of each face and two on bottom. The top sensor is higher then a cockroach, thus in case of a wall, top and bottom sensors will be activated whereas in case of a cockroach, only bottom sensors will be activated. One top and one bottom sensor could have been enough but because of the length and the width of the robot and the sensor's opening angle it would have been difficult to perceive obstacles. Moreover, the robot also needs to make a difference between another InsBot and a wall. In this case we will use local communication with the IR sensors. The nearby robot will emit IR signals whereas the border of the arena will not.

3) Linear Camera: The linear camera will be useful to detect objects or group of cockroaches at a higher distance then the IR sensors. Dark spots will be assumed as group of individuals.

4) Temperature: Temperature information could be used to adapt the behaviour depending on the temperature if needed but we will most probably use it to follow a temperature gradient like cockroaches, that's why we have implemented two of them placed on the extreme positions of the top of the InsBot.

\section{Control and electronics}

In our project there are many different aspects and many different abstraction layers, indeed there is a behaviour level managed by biologist and a hardware low level for engineers. As we will both work on the robot, we finally decided to include two separate processors, also because of the number of peripherals and the necessary computational power (Fig. 8). The first one called "Hardware Processor" is connected to most of the hardware resources (wireless communication, IR sensors and motors); its basic tasks are to control all this features. This processor will be programmed by the engineers. The second one called "Behaviour Processor" will access to all resources through a fast $\mathrm{I} 2 \mathrm{C}$ bus $(400 \mathrm{KHz})$ but can also be interrupted by the "Hardware Processor" with IOs.

The camera is the only hardware device which is connected to the "Behaviour Processor" because of the limited number of IOs on the other processor but mainly because in such a way the information is ready (no need to use the $I^{2} C$ bus) for the high level algorithms.

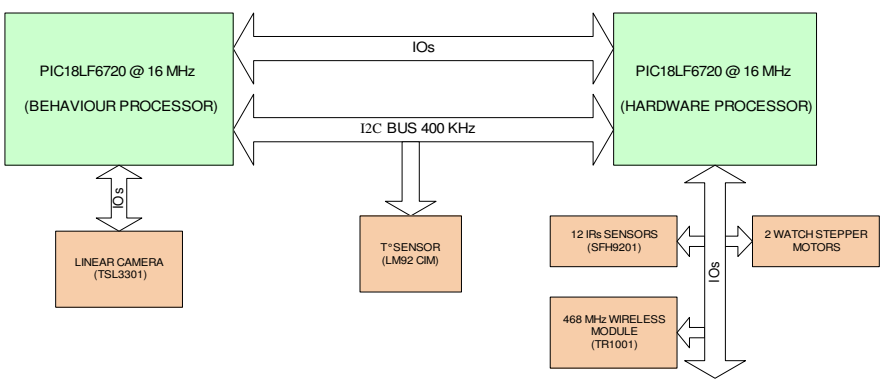

Fig. 8. Control and electronic architecture with 2 microcontrollers.

\section{Energy}

Energy is delivered by a small Li-Polymer 190mAh battery for up to 4 hours autonomy in the worst case when all features are permanently working. We chose Li-Polymer technology because this has currently the highest volumetric capacity, it is available on the market, has affordable price and has a very fast charging time (1 hour for a fast full charge).

\section{E. Mechanics}

The robot must be very small with many features, that's why we had nearly no space for any mechanical part. So we had imagined a new design based only on printed circuit boards (PCB). So each face of the robot is a PCB and connections between each PCB are soldered for both electrical and mechanical connection (Fig. 9).

About locomotion, as for the Alice robot, we chose a differential drive configuration which is non-holmic but still allows most of the displacements by controlling the two motors independently.

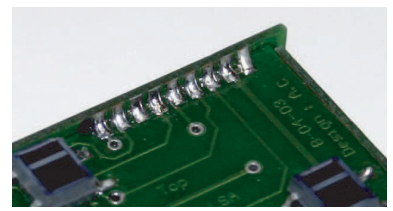

Fig. 9. Soldered electrical and mechanical connections.

\section{F. 3D Drawing}

Because of the very small size and the high integration level, we have first developed a 3D model of the InsBot (Fig. 10) to study the size and the position of each PCB. The 3D model is also very important to visualize the position of each sensor.
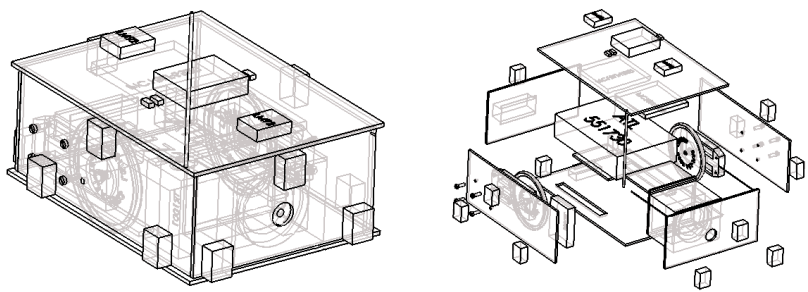

Fig. 10. 3D Model of InsBot: transparent view (left) and exploded view (right).

\section{IMPLEMENTATION}

After design and modelling, we have finally built an InsBot to validate all assembly aspects. The robot is composed by 9 PCBs (0.6 mm thick) as shown on Fig. 11 and TABLE II. The first prototypes have been manually assembled but because of the very small size of all components and the very precise mechanical parts, it takes around 6 hours to fully assemble one InsBot. We planned to build 30 robots for all experiments, thus we will be forced to outsource at least the mounting and soldering of the components.

TABLE III resumes the general specifications of the InsBot. 


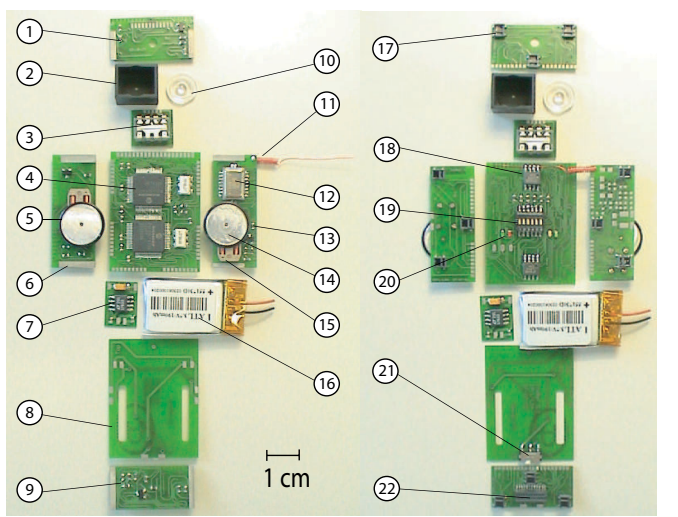

Fig. 11. Disassembled InsBot with all parts: left the PCB seen from inside and right the PCB seen from outside of the robot.

TABLE II

COMPONENT LIST DEPICTED ON FIG. 11

\begin{tabular}{|ll|ll|}
\hline 1. & Front PCB & 12. & HF module $468 \mathrm{MHz}$ \\
2. & Camera box & 13. & Right board \\
3. & Linear camera sensor & 14. & Wheel \\
4. & $2 \times$ PIC18LF6720 processors & 15. & Watch stepper motor \\
5. & Tyre (annular ring) & 16. & Li-Polymer 190mAh battery \\
6. & Left PCB & 17. & $12 \times$ IR sensors \\
7. & 3V regulator & 18. & $2 \times$ High precision T sensors \\
8. & Bottom PCB & 19. & $6 \times$ switches \\
9. & Rear PCB & 20. & $4 \times$ LEDs \\
10. & Plastic lens & 21. & General interrupter (on-off) \\
11. & HF antenna & 22. & General connector \\
\hline
\end{tabular}

TABLE III

SPECIFICATIONS

\begin{tabular}{|ll|}
\hline Weight: & $15 \mathrm{~g}$ \\
Maximum speed: & $5 \mathrm{~cm} / \mathrm{s}$ \\
Autonomy: & 4 hours minimum \\
Operating voltage: & $3 \mathrm{~V}$ \\
Linear camera: & 102 pixels, 8 bits grey level \\
Processor: & $2 \times$ PIC18LF6720 (64Ko Flash) @ $16 \mathrm{MHz}$ \\
T sensors: & $0.33 \mathrm{C}$ accuracy \\
Proximity sensors: & up to $8 \mathrm{~cm}$ \\
Wireless link: & 125000 baud, 20 meters \\
Size: & $41 \mathrm{~mm}(\mathrm{~L}) \times 30 \mathrm{~mm}(\mathrm{~W}) \times 19 \mathrm{~mm}(\mathrm{H})$ \\
Cost: & $200 \mathrm{USD}$ (without assembly) \\
\hline
\end{tabular}

\section{SOFTWARE}

Source codes of the InsBots has been written in $\mathrm{C}$ and compiled with CCS PCW compiler (http://www.ccsinfo.com). The compiled hex file is then uploaded in the robot thanks to a serial bootloader, so user doesn't need any hardware programmer.

TABLE IV is an abstract of the software architecture of the InsBot.

\section{EXTERNAL TOOLS}

With any robot but much more for small size robots, tools to program, debug and work efficiently are really important.

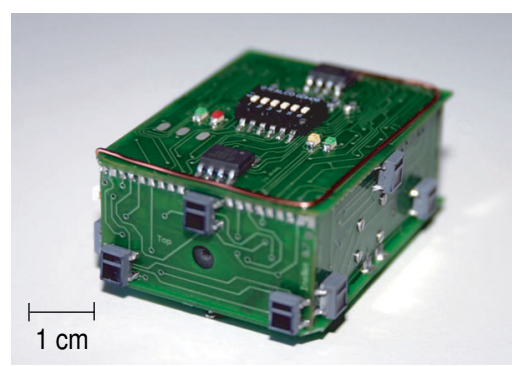

Fig. 12. InsBot fully assembled.

TABLE IV

SOFTWARE ARCHITECTURE

\begin{tabular}{|l|l|}
\hline Software processor & Hardware processor \\
\hline No OS, library of functions & Multitask and real-time OS \\
\hline Random generators & Motors control \\
- Uniform & Sensors processing : \\
- Normal & - Proximity \\
- Exponential & - Brightness \\
Hardware access & Time in milliseconds (4 Bytes) \\
Hi-level behaviours & Automatic behaviours : \\
& - Obstacle (avoidance-attraction) \\
& - Wall following (left-right) \\
& - Light (avoidance-attraction) \\
& - Temperature (avoidance-attraction) \\
& Local communication (IR) \\
& Global communication (HF) \\
\hline
\end{tabular}

That's why in parallel of the InsBot development, we have developed different external helping tools.

\section{A. Programming Board}

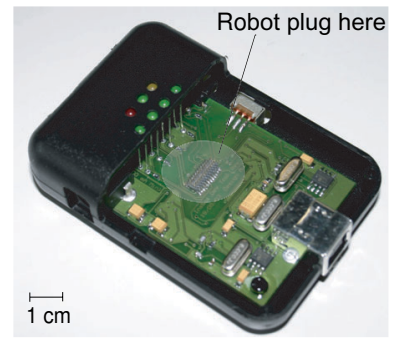

Fig. 13. Programming and charging board.

Each InsBot processor can communicate with a PC through a serial port (also used to reprogram) using the programming board (Fig. 13). In most of the actual laptop PC there are no serial ports that's why we decided to implement a USB hub and two USB-Serial converters so that it needs just one USB Port on the PC.

It also includes ICD (In-Circuit Debugging) connections for a full re-flash of the memory. The last feature that has been included is a fast charger (full battery charge in 1 hour).

\section{B. Charger block}

As we planned to work with colonies of 20 individuals, we have developed a charger block (Fig. 14) which includes the same charger as the programming board, but it permits 


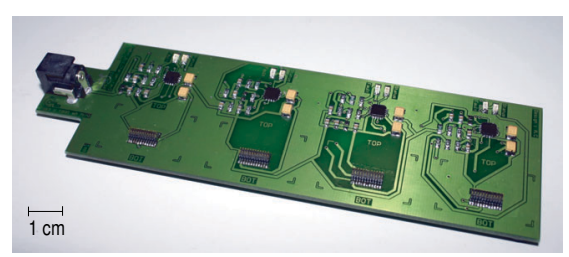

Fig. 14. Fast charger for four InsBots

recharging 4 InsBots at the same time. It also monitors the battery charging level and other problems. The state is signalized with two LEDs.

\section{Radio base station}

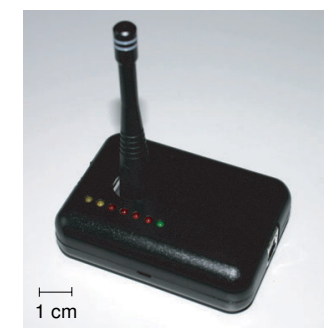

Fig. 15. Wireless base board

To communicate with the InsBot, a serial cable can be used but also the wireless link. Thanks to this board (Fig. 15) we can communicate both with one InsBot, defined by its address, or with all InsBots. The robots can also communicate together. As said before we will not use this wireless link to globally control the behaviour of all the InsBot, but only for monitoring the experiments. The board uses a USB-Serial converter to be compatible with any computer (Windows, MacOs and Linux), and thanks to the USB power this module doesn't require any external power supply. All existing applications communicating using a classical serial port (MatLab, SysQuake, Hyperterminal, etc.) are also compatible because the USBSerial converter emulates a virtual serial port on the computer.

\section{FUTURE WORK}

We are now working on the behaviours to be implemented in the InsBot in order to mimic the insects. First the biologists will extract the trajectories of the cockroaches using the tracking system and will use these in order to find all the parameters of the individual behaviour model. The first goal is to have a robot that behaves like an individual cockroach. In order to prove it we will try a Turing test on the trajectometry of the newly programmed robot. After this first phase, we will introduce the robot in the colony and we will try to analyze which are the most important parameters (size, noise, behaviour, chemistry) that enable the InsBots to be recognized as a congener. The last part will be to upgrade the individual behaviour of the InsBots to influence the global behaviour of the mixed society. The typical experiment in this sense may be to attract the cockroaches in a place they would not go without the presence of the robot. At this point it will be possible to study and test the parameters and the strategies that permit us to control the whole group.

\section{CONCLUSION}

After around 6 months of analysis with an existing mini robot (Alice), cooperating with biologists, we have been able to define specifications for a new robot to be used in a mixed society together with cockroaches. Because of the limitation on the physical dimensions and the large number of necessary sensors, some tradeoffs have been decided. However, this robot has been designed, realized and tested. The number of features included in the robot called InsBot makes it an example of highly integrated system. Moreover, this is one of the first robots devoted to interaction with small insects. It is an indispensable step in order to understand mechanisms in complex societies of social animals and will hopefully give a possibility to even control such mixed societies.

\section{ACKNOWLEDGMENT}

Many thanks to André Guignard from EPFL for the moulded part (linear camera).

The LEURRE project is funded by the Future and Emerging Technologies programme (IST-FET) of the European Community, under grant IST-2001-35506. The information provided is the sole responsibility of the authors and does not reflect the Community's opinion. The Community is not responsible for any use that might be made of data appearing in this publication. The Swiss participants to the project are supported under grant 01.0573 by the Swiss Government.

\section{REFERENCES}

[1] R.C. Arkin, M. Fujita, T. Takagi, R. Hasegawa, "Ethological Modeling and Architecture for an Entertainment Robot", Proc. ICRA'01, vol. 1, p. 453-458, 2001

[2] N. Kagawa, H. Kazerooni, "Biomimetic Small Walking Machine", Proc. Int. Conference on Advanced Intelligent Mechatronics, AIM'01, vol.2, p. 971-9, 2001.

[3] G.M. Nelson, et al, "Design and Simulation of a Cockroack-Like Hexapod Robot", Proc. ICRA'97, vol. 2, p.1106-1111, 1997.

[4] D. Price, "Climbing the walls: a look at a cockroach-like robot", IEEE Expert, vol. 10, issue 2, p. 67-70, 1995.

[5] H.H. Lund, B. Webb, J. Hallam, "A Robot Attracted to the Cricket Species Gryllus bimaculatus", Proc. European Conference on Artificial Life, ECAL'97, p. 246-255, 1997.

[6] R. Holzer, I. Shimoyama, "Locomotion Control of a Bio-Robotic System via Electric Stimulation", Proc. IROS'97, vol. 3, p. 1514-1519, 1997.

[7] M. Boehlen, "A robot in a cage", Proc. IEEE Int. Symp. on Computational Intelligence in Robotics and Automation, CIRA99, p. 214219, 1999.

[8] R. Vaughan, N. Sumpter, J. Henderson, A. Frost, S. Cameron, "Robot Control of Animal Flocks", Proc. Int. Symp. Intelligent Control, p. $277-$ 282,1998

[9] W.J. Bell and K.G. Adiyodi, American Cockroach, Chapman and Hall Ltd, 1982.

[10] J.M. Amé, C. Rivault, J.L. Deneubourg, "Cockroach aggregation based on strain odour recognition", Animal Behaviour, 2004.

[11] R. Jeanson, et al, "A model of animal movements in a bounded space", Journal of Theoretical Biology 225, p. 443-451, 2003.

[12] E. Bonabeau, M. Dorigo, G. Theraulaz, Swarm Intelligence: From Natural to Artificial Systems, Oxford University Press, Oxford, 1999.

[13] G. De Shutter, G. Theraulaz, J-L. Deneubourg, "Animal-robots collective intelligence", Annals of Mathematics and Artificial Intelligence, vol. 31, p. 223-238, 2001.

[14] G. Theraulaz, J. Gautrais, S. Camazine, J.L. Deneubourg, "The formation of spatial patterns in social insects: from simple behaviours to complex structures", Phil. Trans. Royal Society, A 361, p. 1263-82, 2003.

[15] G. Caprari, T. Estier, R. Siegwart, "Fascination of Down Scaling - Alice the Sugar Cube Robot", Journal of Micro-Mechatronics, vol. 1, no. 3, p. 177-189, 2002 